\title{
Akute pulmonale Infektionen - was ist wann typisch?
}

Akute pulmonale Infektionen sind ein äußerst häufiges Krankheitsbild in der Pädiatrie. In Abgrenzung zu oberen Atemwegsinfekten (Laryngitis, Tracheitis, Bronchitis) werden hier im Folgenden nur akute Pneumonien diskutiert. Die Einteilung der akuten Pneumonie kann verschiedenen Mustern folgen. Leicht zu eruieren ist die Frage der lokalen Umgebung des Erkrankten. Es können nosokomiale Pneumonien oder community acquired pneumonias (CAP), was wesentlich häufiger der Fall ist, vorliegen. Eine weitere Unterscheidung ermöglicht das Fehlen/Vorliegen von Risikofaktoren (anatomische Anomalien, Immundefekte, chronische Grunderkrankungen, z. B. CF, $P(D)$. Auch das Alter der pädiatrischen Patienten und artifizielle Ursachen (z. B. Aspirationspneumonie) sind zusätzliche Unterscheidungsmerkmale. Die Kenntnis dieser Grundlagen erleichtert, da sie z. T. eine typi- sche Anamnese oder einen klinischen Untersuchungsbefund ergeben, diagnostische und auch therapeutische Entscheidungen. Die Unterscheidung verschiedener Erreger aufgrund von Klinik und Röntgen-Thorax ist schwierig.

Eine Unterscheidung zwischen bakterieller und viraler Pneumonie ist ein Puzzle. Einzelne Beobachtungen bzw. Laborwerte sind dabei nur bedingt hilfreich. Für diese und andere Mythen der Diagnostik werden Beispiele erörtert.

\section{Interessenkonflikt}

Die Autoren geben an, dass kein Interessenkonflikt besteht.
Autorinnen/Autoren

M. Ballmann

Universitätsmedizin Rostock, Kinder- und Jugendklinik

\section{Korrespondenzadresse}

M. Ballmann

Universitätsmedizin Rostock, Kinder- und Jugendklinik

manfred.ballmann@med.uni-rostock.de

\section{Bibliografie}

DOI https://doi.org/10.1055/a-0943-0945 Online-Publikation: 2019

Fortschr Röntgenstr 2019; 191: S105

(c) Georg Thieme Verlag KG, Stuttgart · New York ISSN 1433-5972 